\title{
Temporal primary cutaneous carcinosarcoma in the ear: A case report
}

\author{
Issam Msakni ${ }^{1,3}$, Aya Khemir ${ }^{1,3}$, Nada Mansouri ${ }^{1,3}$, Karima Tlili $^{1,3}$, Faten Gargouri ${ }^{1,3}$, \\ Ghassen Chebbi ${ }^{2,3}$, Besma Laabidi ${ }^{1,3}$
}

${ }^{1}$ Pathology Department, Military Hospital of Tunis, Tunisia, ${ }^{2}$ Otorhinolaryngology Department, Military Hospital of Tunis, Tunisia, ${ }^{3}$ Faculty of Medicine of Tunis, University of Tunis El Manar, Tunis, Tunisia

Corresponding author: Dr. Issam Msakni, E-mail: msaknissam@yahoo.fr

\begin{abstract}
Carcinosarcoma is a rare biphasic tumor made of two malignant components-the epithelial component and the stromal component - that can develop anywhere on the body, but mainly in sun-exposed areas. We report the case of a 78-year-old male who presented himself with a right temporal tumor in the ear $10 \mathrm{~cm}$ in diameter. A biopsy suggested a sarcoma. The patient underwent an extensive surgical resection of the temporal mass and the ipsilateral ear. A microscopic examination of the tumor revealed two intermixed malignant contingents. The epithelial component was made of atypical basaloid cells arranged in nests with peripheral palisading and expressing cytokeratin. The stromal component was made of atypical spindle cells expressing smooth muscle actin (SMA). A diagnosis of primary cutaneous carcinosarcoma with clear margins was reached. The patient is alive and without recurrence after twelve months of a follow-up period.
\end{abstract}

Key words: Cutaneous carcinosarcoma; Metaplastic carcinoma; Prognosis

\section{INTRODUCTION}

Carcinosarcoma is a rare biphasic tumor made of two malignant components-the epithelial component and the stromal component [1-5] —also referred to as metaplastic carcinoma [6,7], with a male-to-female predominance. Carcinosarcoma affects elderly patients, appearing anywhere on the body [1,4-7]. The first case of cutaneous carcinosarcoma (CC) was described in 1972 [8]. CC occurs in the head and neck, but mainly in the face, scalp [1], forearms, and all other sun-exposed areas of the body [7]. The tumor can grow slowly or increase rapidly in size [5]. The current recommended treatment of $\mathrm{CC}$ is complete surgical excision [1,9]. Its prognosis is poorly defined given its rarity: only 120 cases of CC have been reported in the literature [7]. We report a new case of a primary $\mathrm{CC}$ in a 78-year-old male patient.

\section{CASE REPORT}

A 78-year-old male presented himself with a right temporal tumor mass. A physical examination revealed an ulcerative and infiltrative tumor mass, large and extensive, eroding the right ear below. The physical examination was otherwise normal and there was no notable lymphadenomegaly. An imaging survey was normal outside the temporal tumor. Laboratory tests were within normal limits as well. The tumor mass was biopsied. A pathological examination led to the diagnosis of sarcoma. Therefrom, the patient underwent extensive surgical excision of the tumor and the right ear. A gross examination showed an ulcerative tumor, $10 \mathrm{~cm}$ in diameter, infiltrating the underlying skin layers. The right ear was eroded by the tumor in its upper portion. A large sampling was made, and a microscopic examination revealed a biphasic malignant proliferation. The tumor was made of two components: an epithelial and a mesenchymal. The epithelial component was made of nests of atypical basaloid cells with peripheral palisading (Fig. 1), reminiscent of the features of basal cell carcinoma. The nests were intermixed with the sarcomatous component, which was made of atypical spindle cells organized in a storiform pattern (Fig. 2). Immunohistochemical

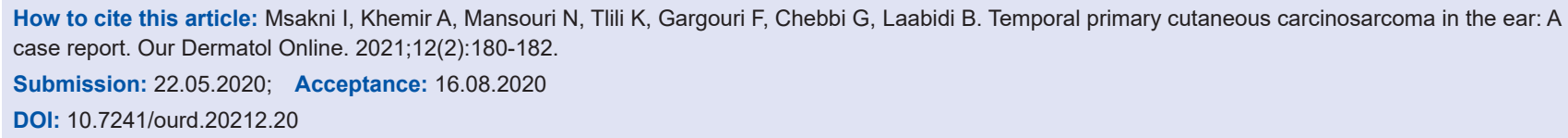




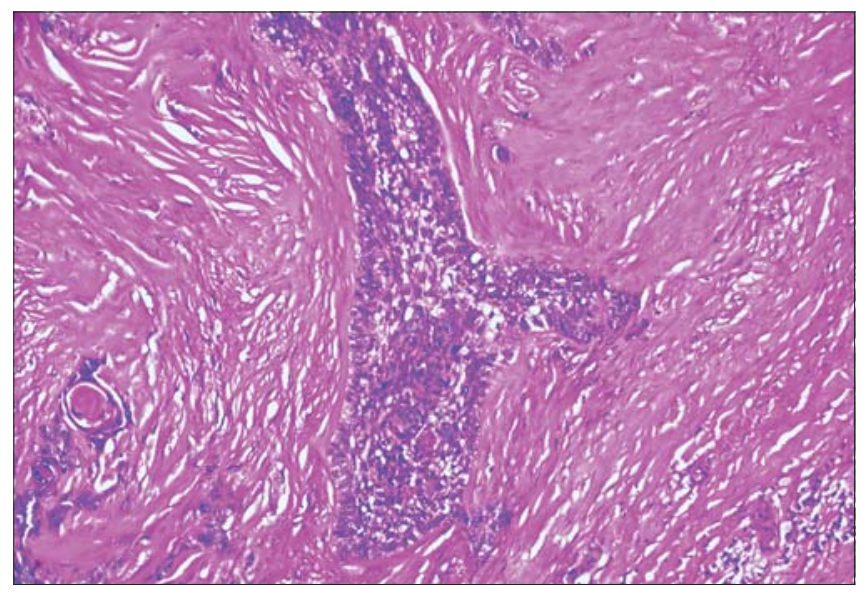

Figure 1: Atypical basaloid cells in the epithelial contingent arranged in cords, showing peripheral palisading reminiscent of the features of basal cell carcinoma (H\&E, 10x).

staining showed a positivity of the tumor cells in the epithelial contingent for cytokeratin and a negativity for epithelial membrane antigen (EMA) and smooth muscle actin (SMA). The mesenchymal contingent showed a diffuse positivity for SMA and cytokeratin (Fig. 3) and a negativity for EMA.

The diagnosis of primary cutaneous basal cell carcinosarcoma was upheld. Surgical margins were clear. The patient is alive without recurrence after a 12-month follow-up period.

\section{DISCUSSION}

Carcinosarcoma is a rare biphasic tumor [1-5]. The term carcinosarcoma was first proposed in 1864 [10]. Carcinoma affects elderly patients $[1,5-7]$ and may develop anywhere on the body: in the skin, uterus, lung, or digestive tract $[1,4,5]$. The first case of CC was described in 1972 [8]. As far as we know, only 120 cases of CC have been reported in the literature [1]. $\mathrm{CC}$ occurs in the head and neck, but mainly in the face and scalp, as in our case [1], as well as in the forearms and other sun-exposed area of the body [7]. The tumor can grow slowly or increase rapidly in size [5]. Its development in sun-exposed areas explains the existence of squamous dysplasia in areas adjacent to the carcinosarcoma, reminiscent, thus, of sun-induced skin damage [1]. The presence of such lesions may help if a metastatic origin is suspected. CC can be secondary to a carcinosarcoma of the gastrointestinal tract or bones. The skin is an infrequent site for gastrointestinal or bone cancer metastases but makes prognosis difficult if affected.

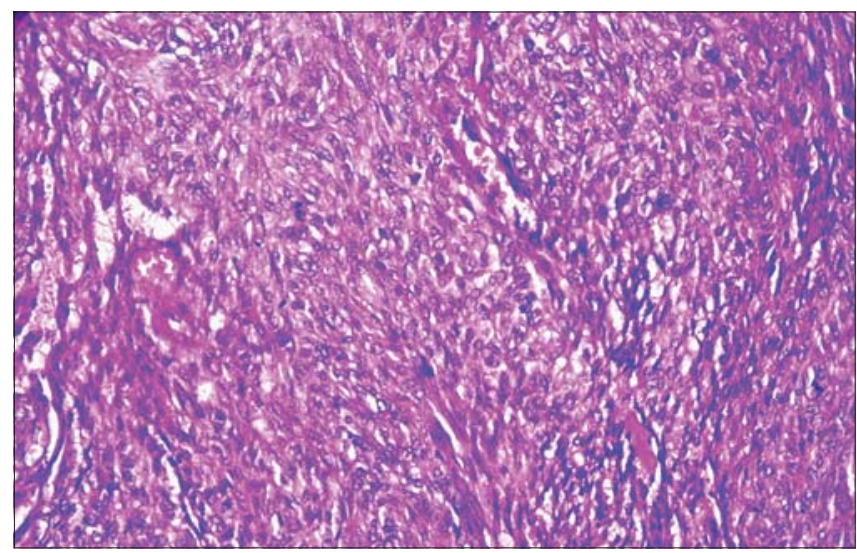

Figure 2: Atypical spindle cells in the stromal contingent arranged in a storiform pattern (H\&E, 10x).

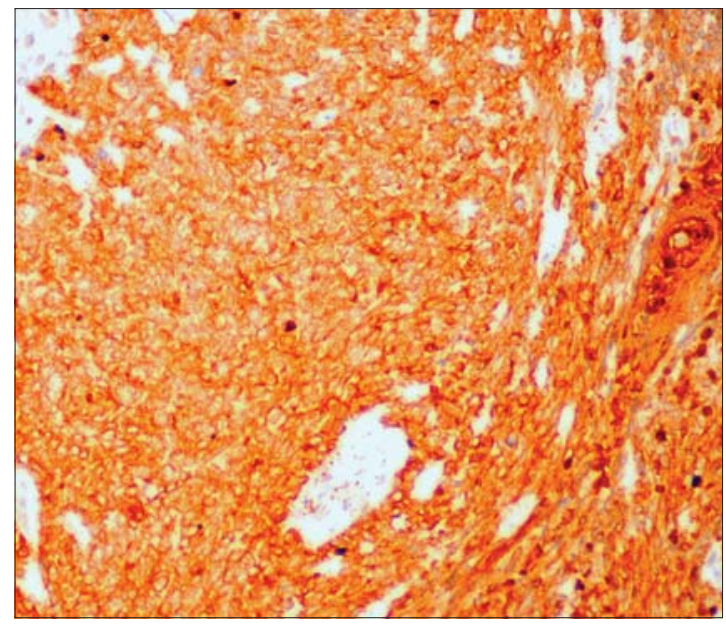

Figure 3: Tumor cells in the stromal component with a marked diffuse positivity for cytokeratin antibody (H\&E, 10x).

Morphologically, the tumor is biphasic and made of two adjacent or intermixed malignant components: the epithelial component and the heterologous stromal component [1]. Due to its heterogeneity, such a tumor necessitates large sampling not to misdiagnose it simply as a carcinoma or sarcoma. Cases of CC misdiagnosed as the spindle cell variant of squamous cell carcinoma or atypical fibroxanthoma due to insufficient sampling have been reported. Besides, it can be confused with differentials such as leiomyosarcoma, fibrosarcoma, and other mesenchymal malignancies, especially if the stromal component is predominant, as in the case of our patient [1].

The pathogenesis of carcinosarcoma has not yet been well understood [2]. The same chromosomal changes have been identified in both the stromal and epithelial components [7]. A study by Kwak et al. reviewed 11 cases of carcinosarcoma in Korean patients. The epithelial component stained positively for epithelial markers in 
all cases. The stromal component stained positively for epithelial markers in $63 \%$ of cases, as in our study, where the stromal contingent stained positively for cytokeratin [1]. These results were in favor of the dated hypothesis suggesting that the stromal component is a divergence of the epithelial component [7].

Surgical excision is the current recommended treatment for CC, but there are no well-defined sufficient surgical margins reported in the literature [1,9]. Excised tumors recur often [3].

Given the rarity of CC, its prognosis is poorly known [7]. However, a relatively better prognosis for the group of basal cell carcinosarcomas has been reported [3]. A study by Tran compared two groups of patients diagnosed with CC. The subdivision into groups was according to the histological type derivation of the epithelial component. In the first group, the epithelial component was of the epidermal type (squamous or basal malignant component). In the second group, the epithelial component was of the adnexal type (including spiradenocarcinoma and porocarcinoma). The conclusion was that the patients from the first group were older and had a better prognosis. In contrast, the patients from the second group were younger but a had poorer prognosis $[5,6]$.

$\mathrm{CC}$ is a rare and aggressive malignancy that develops in sun-exposed areas of the body, affecting elderly male patients. Cases of $\mathrm{CC}$ reported in the literature appeared mostly in the head and neck. Its pathological diagnosis is challenging because of its rarity. It can easily be misdiagnosed as a sarcoma or carcinoma especially due to insufficient sampling. In some cases, and despite large sampling, the diagnosis of $\mathrm{CC}$ remains challenging because one of the two malignant contingents-either epithelial or stromal—may be predominant and conceal the other, for which immunohistochemical staining may be of great help. Surgical excision with negative margins is recommended to prevent recurrences. The prognosis is not yet well-defined, given the limited number of cases studied in the literature so far.

\section{Consent}

The examination of the patient was conducted according to the principles of the Declaration of Helsinki.

The authors certify that they have obtained all appropriate patient consent forms, in which the patients gave their consent for images and other clinical information to be included in the journal. The patients understand that their names and initials will not be published and due effort will be made to conceal their identity, but that anonymity cannot be guaranteed.

\section{REFERENCES}

1. Kwak HB, Park J, Kim HU, Nam KH, Yun SK. Cutaneous carcinosarcoma: a clinicopathologic and immunohistochemical analysis of 11 Korean cases. J Korean Med Sci. 2019;34:e5.

2. Daisley Jr. D, Trim S, Daisley AR. Caesarean section scar endometriosis: a case report and review of the literature with special emphasis on malignant transformation. Our Dermatol Online. 2018;9:176-9.

3. Sinha A, Houghton D, Beer T, Yu L, Wood BA. Basal cell carcinosarcoma: a report of three cases and review of the literature. Pathology. 2013;45:S114.

4. Clark JJ, Bowen AR, Bowen GM, Hyngstrom JR, Hadley ML, Duffy K, et al. Cutaneous carcinosarcoma: a series of six cases and a review of the literature. J Cutan Pathol. 2017;44:34-44.

5. Suzuki H, Hashimoto A, Saito R, Izumi M, Aiba S. A case of primary cutaneous basal cell carcinosarcoma. Case Rep Dermatol. 2018;10:208-15.

6. Tran TA, Muller S, Chaudahri PJ, Carlson JA. Cutaneous carcinosarcoma: adnexal vs. epidermal types define high- and lowrisk tumors. Results of a meta-analysis. J Cutan Pathol. 2005;32:2-11.

7. Messina J, Epstein EH Jr, Meckenzie C, Kossard S, Patel RM, Patterson JW, et al. Basal cell carcinoma. In: Classification of skin tumors. 4th ed. Lyon: David E. Elder, Daniela Massi, Richard A. Scolyer, Rien Willemze; 2018. p. 26-34.

8. Ruiz-Villaverde R, Aneiros-Fernández J. Primary cutaneous carcinosarcoma: a cutaneous neoplasm with an exceptional presentation. Sultan Qaboos Univ Med J. 2018;18:e114-5.

9. El Harroudi T, Ech-Charif S, Amrani M, Jalil A. Primary carcinosarcoma of the skin. J Hand Microsurg 2016;2:79-81.

10. Iakovides J, Delides GS. Carcinosarcomas of the skin--report of two cases. Arch Geschwulstforsch. 1988;58:461-4.

Copyright by Issam Msakni, et al. This is an open-access article distributed under the terms of the Creative Commons Attribution License, which permits unrestricted use, distribution, and reproduction in any medium, provided the original author and source are credited.

Source of Support: Nil, Conflict of Interest: None declared. 